\title{
Computational study of wave propagation through a dielectric thin film medium using WKB approximation
}

\author{
Emmanuel Ifeanyi Ugwu', Sadik Olaniyi Maliki ${ }^{2}$ and Michael Alo Elebe ${ }^{1}$ \\ ${ }^{1}$ Department of Industrial Physics and ${ }^{2}$ Department of Industrial Mathematics and Applied \\ Statistics; Ebonyi State University, Abakaliki, Nigeria.
}

\begin{abstract}
Wave propagation in a non-homogeneous dielectric thin film medium using W.K.B approximation technique is presented. The approximation is used to solve a scalar wave equation that contains a dielectric perturbation, $\Delta \varepsilon_{p}(z)$ by imposing all the W.K.B approximation conditions in conjunction with other necessary boundary conditions that were applied in the solution. The influence of the perturbation on the propagating wave profile through the thin film for ultra-violet, optical and infrared regions of electromagnetic wave was analyzed.
\end{abstract}

Keywords: Thin film, wave propagation, W.K.B approximation, dielectric perturbation, wave profil,e non-homogeneous medium, scalar wave equation

\section{INTRODUCTION}

Different methods and approach have been used in the study of wave propagation in an inhomogeneous medium such as wave guide, thin film etc [Ugwu, 2011, Ong and Meyer, 1983] notwithstanding the complication and rigor involved in approach, it is interesting because of its applicability in the study of material and nanocsiences. Abele's $2 \times 2$ matrix method which is a scheme used for computation of the optical effect in a layered media had been studied [Abeles,1950] . In addition, geometrical approximation, phase integral method, generalized geometrical optic approximation and method of perturbation theory have also been used to study propagation in non-homogeneous media[Thyle'n1983]This was enhanced because the approach permits an understanding and unified treatment of guided radiation mode and also permits the propagation of a given field in rather general refractive index structure. Apart from permitting an understanding and assessment of various properties of the propagated field, the method can also again be used in computing the propagating constant [Thyle' $n$ et al1992; Feit and Fleck 1978] Wentzel-KramersBrillouin, W.K.B approximation technique has been one of those approximation that has been used in studying propagation through non-uniform media both classically and quantum mechanically, especially when it has got to do with scattering problems[Davydov,1991;Fitzpartrick,2002;Ugwu,200

9] Generally, as it is clearly known that when wave propagates either normally or obliquely incident on a surface or interface where the refractive index exhibit a sudden change within the medium, there is generally an appreciable observed reflection of wave [Ugwu et al 2007] especially in a situation where W.K.B approximation technique which is an approximate solution obtained by considering that the incident propagating wave through the medium experiences slowly varying refractive index which on the other hand introduces a change in the wavelength of the propagating wave. This change for sure brings about a change in the phase of the wave which the use of an approximation can be made possible introducing the concept of Jeffries connection formula which enables one to compute the reflection co-efficient, a concept that favours a proper understanding of the reflection properties of the material medium of the thin film material [Ugwu and Uduh 2005] Though it is certain that many of these approximation technique being used in the study of wave propagation through an inhomogeneous medium involved formulation which has shortcoming [Thyle'n et al,1983] as it always made use of neglecting some factors, it can be improved upon by applying some certain correction terms to the approximation [ Feit and Fleck, 1980]. In this paper we consider the wave propagation through a dielectric thin film medium using W.K.B approximation method with view of analyzing the influence of the dielectric perturbation, $\Delta \varepsilon_{p}(z)$ on the propagating field profile through the thin film 


\section{Reference Medium}

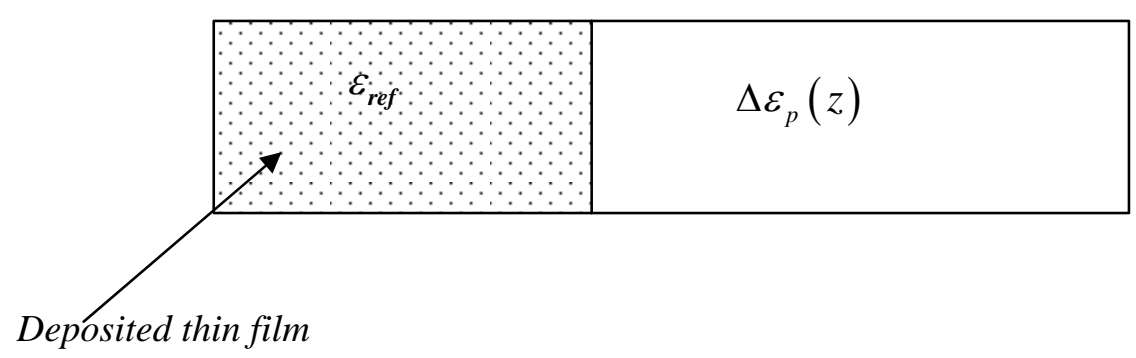

Fig.1 Deposited thin film model on a substrate

\section{THEORETICAL PROCEDURE}

\section{Given}

$\psi^{\prime \prime}(z)+\omega^{2} \mu_{0} \varepsilon_{p}(z) \psi(z)=0$

with

$\varepsilon_{p}(z)=\Delta \varepsilon_{p}(z)+\varepsilon_{r e f}$

Where $\Delta \varepsilon_{p}(z)$ is the dielectric perturbation defined on the thin film medium and $\varepsilon_{\text {ref }}$ the dielectric reference medium associated with an empty space without thin film. If we substitute equation (2) into equation (1) we obtain

$$
\begin{array}{r}
\psi^{\prime \prime}(z)+\omega^{2} \mu_{0} \Delta \varepsilon_{p}(z) \psi(z) \\
=-\omega^{2} \mu_{0} \varepsilon_{r e f} \psi(z)
\end{array}
$$

Setting $k_{0}=\omega^{2} \mu_{0}$, then we have;

$\psi^{\prime \prime}(z)+k_{0} \Delta \varepsilon_{p}(z) \psi(z)=-k_{0} \varepsilon_{r e f} \psi(z)$

Defining $k_{0} \Delta \varepsilon_{p}(z)=-V(z)$ as a term containing the perturbation, and neglecting the right hand side, equation (3) becomes;

$\psi^{\prime \prime}(z)-V(z) \psi(z)=0$

We will assume equation (4a) to have a solution of the form

$\psi(z)=\exp (i \phi(z))$

Differentiating twice, we obtain

$\psi^{\prime \prime}(z)=i \phi^{\prime \prime} \exp (i \phi)-\left(\phi^{\prime}\right)^{2} \exp (i \phi)$

$$
\begin{aligned}
& i \phi^{\prime \prime} \exp (i \phi)-\left(\phi^{\prime}\right)^{2} \exp (i \phi) \\
&-V(z) \exp (i \phi)=0
\end{aligned}
$$

Assume $\phi^{\prime \prime}$ to be very small, and since $\exp (i \phi) \neq 0$ then

$$
\begin{aligned}
& \left(\phi^{\prime}\right)^{2}=V(z) \Rightarrow \phi^{\prime}= \pm i \sqrt{V(z)} \\
& \phi(z)= \pm \int \sqrt{V(z)} d z
\end{aligned}
$$

In accordance with WKB approximation, the condition for $\phi^{\prime \prime}$ to be considered very small results from the fact that;

$\left|\phi^{\prime \prime}\right| \approx \frac{1}{2}\left|\frac{V^{\prime}}{\sqrt{V}}\right| \ll|V|$

A second approximation is found by iteration from equation (7) which gives us

$\left|\phi^{\prime \prime}\right| \approx \pm \frac{1}{2} V^{-\frac{1}{2}} V^{\prime}$

substituting into equation (6) gives

$\psi(z) \approx \frac{1}{\sqrt[4]{-V(z)}}\left\{\begin{array}{c}c_{1} \exp \left(i \int \sqrt{-V(z)} d z\right) \\ +c_{2} \exp \left(-i \int \sqrt{-V(z)} d z\right)\end{array}\right\}$

$\psi(z) \approx \frac{1}{\sqrt[4]{-V(z)}}\left\{\begin{array}{c}c_{1} \exp \left(-\int \sqrt{V(z)} d z\right) \\ +c_{2} \exp \left(\int \sqrt{V(z)} d z\right)\end{array}\right\}$ 
The factor $\frac{1}{\sqrt[4]{-V(z)}}$ will be taken simply as a constant and therefore can be embedded in the constants $C_{1}$ and $C_{2}$ to give new constants $A$ and $B$. Thus the approximate solution can be written

$$
\begin{aligned}
\psi(z) \approx A \exp (- & \left.\int \sqrt{V(z)} d z\right) \\
+ & B \exp \left(\int \sqrt{V(z)} d z\right)
\end{aligned}
$$

Now

$$
\begin{aligned}
\int \sqrt{V(z)} d z & =\int \sqrt{-k_{0}^{2} \Delta \varepsilon_{p}} d z \\
& =i \frac{2 \pi z}{\lambda} \sqrt{\Delta \varepsilon_{p}}
\end{aligned}
$$

Hence the required solution is

$$
\begin{aligned}
\psi(z) \approx A & \exp \left[-i\left(\frac{2 \pi \sqrt{\Delta \varepsilon_{p}}}{\lambda}\right) z\right] \\
+ & B \exp \left[i\left(\frac{2 \pi \sqrt{\Delta \varepsilon_{p}}}{\lambda}\right) z\right]
\end{aligned}
$$

For large values of $\mathrm{z}$ the term;

$$
\exp \left[i\left(\frac{2 \pi \sqrt{\Delta \varepsilon_{p}}}{\lambda}\right) z\right]
$$

will cause the solution to be unbounded. Hence the correct approximate solution is

$$
\begin{aligned}
\psi(z) \approx A \cos \left(\frac{2 \pi \sqrt{\Delta \varepsilon_{p}}}{\lambda}\right) z \\
+A^{\prime} \sin \left(\frac{2 \pi \sqrt{\Delta \varepsilon_{p}}}{\lambda}\right) z
\end{aligned}
$$

With $\psi(0)=0$, and $\psi^{\prime}(0)=1$ we get; $A=0$,

$$
\therefore \quad \psi(z) \approx A^{\prime} \sin \left(\frac{2 \pi \sqrt{\Delta \varepsilon_{p}}}{\lambda}\right) z
$$

Now

$$
\psi^{\prime}(z)=A^{\prime} \frac{2 \pi \sqrt{\Delta \varepsilon_{p}}}{\lambda} \cos \left(\frac{2 \pi \sqrt{\Delta \varepsilon_{p}}}{\lambda}\right) z
$$

Hence

$$
\begin{aligned}
& \psi^{\prime}(0)=1 \quad \Rightarrow \quad A^{\prime} \frac{2 \pi \sqrt{\Delta \varepsilon_{p}}}{\lambda}=1 \\
& \therefore \quad A^{\prime}=\frac{\lambda}{2 \pi \sqrt{\Delta \varepsilon_{p}}}
\end{aligned}
$$

It then follows that

$$
\psi(z) \approx \frac{\lambda}{2 \pi \sqrt{\Delta \varepsilon_{p}}} \sin \left(\frac{2 \pi \sqrt{\Delta \varepsilon_{p}}}{\lambda}\right) z
$$

gives WKB approximate solution.

\section{RESULT AND DISCUSSION}

The study of the field propagation through a dielectric thin film in this work emanated from the solution of the scalar wave equation on thin film considered to have dielectric perturbation with propagation distance in accordance with the equation(1)as defined in fig. 1 on which WKB approximation technique and other necessary boundary conditions were applied to obtain the solution of the field profile as it propagate through the thin film as in equation (13) The graphs as obtained from equation (13) are shown in fig. 2 depicts the wave profile for three regions of electromagnetic wave comprising ultraviolet ,optical and near infrared region when the dielectric perturbation, $\Delta \varepsilon_{p}(z)=0.50$, $\psi_{1}(z), \psi_{2}(z) a n d \psi_{3}(z)$ represent the profile in each case respectively. From this, it is observed that just for a small propagation distance between 0 to 20 $\times 10^{2} \mathrm{~nm}$ from the thin film, the field profile appear to exhibit the same pattern after which uv profile, $\psi_{1}(z)$ rises sharply and peaked at $80 \times 10^{2} \mathrm{~nm}$ and falls while the optical field profile $\psi_{2}(z)$ and that of infrared $\psi_{3}(z)$ tend to oscillate out of phase as they propagate through the film. Fig. 3 presents the profile when $\Delta \varepsilon_{p}(z)=3.50$ for the same three defined electromagnetic wave regions. In this case, the profile seemed to maintain the same pattern as in the first case but after the propagation distance of 20 $\times 10^{2} \mathrm{~nm} \psi_{1}(\mathrm{z})$ assumes a more linear characteristic while the Optical and infrared profiles ceases and reduces oscillation respectively. In fig. 4 were shown in the profile when, $\Delta \varepsilon_{p}(z)=10.5$ for the same regions in question. Here, the linearity of the uv 
profile becomes more pronounced while $\psi_{2}(z)$ tend to assume a straight line with ceasure of $\psi_{3}(z)$ oscillation. However, this difference in the profiles of the electromagnetic wave regions is due to their difference in response to the influence of scattering and reflection of the propagating wave through the thin film particles. This is because for some of the regions, the small scattered part of their components may combine constructively with the primary incident wave resulting in the phase change that is tantamount to change of the wave velocity propagating through the thin film medium. On the other hand the components may be lost as a result of destructive interference and hence leads to decrease in characteristic wave profile [Brykhovestkii and Tigrov, 1985]

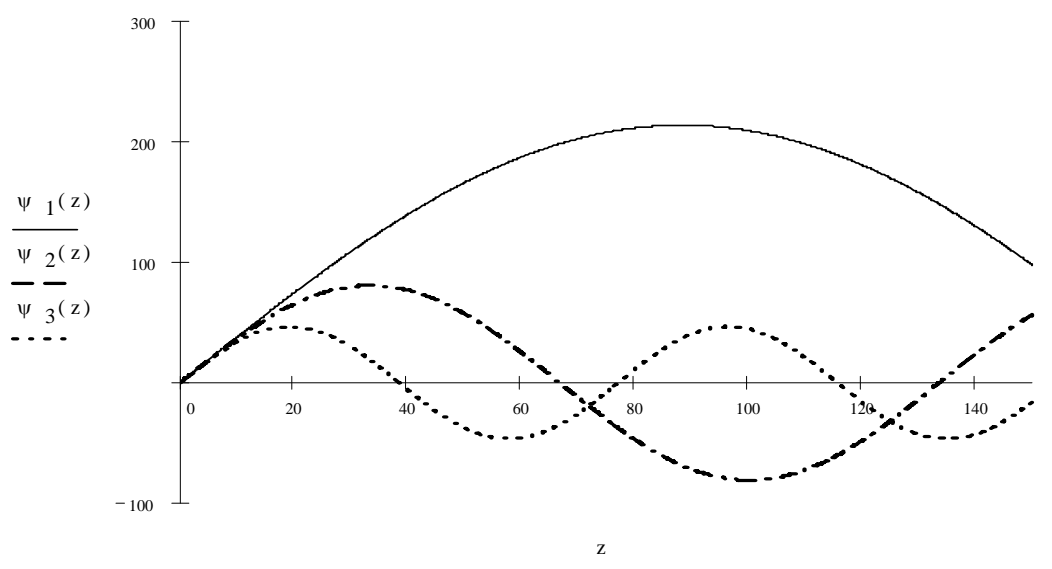

Fig. 2 Field profile in the thin film as it propagates through the thin film thickness $\left(z \times 10^{2} \mathrm{~nm}\right.$ for $\Delta \varepsilon_{p}(z)=0.50$ when $\lambda=250 \mathrm{~nm}, 650 \mathrm{~nm}$ and $950 \mathrm{~nm}$

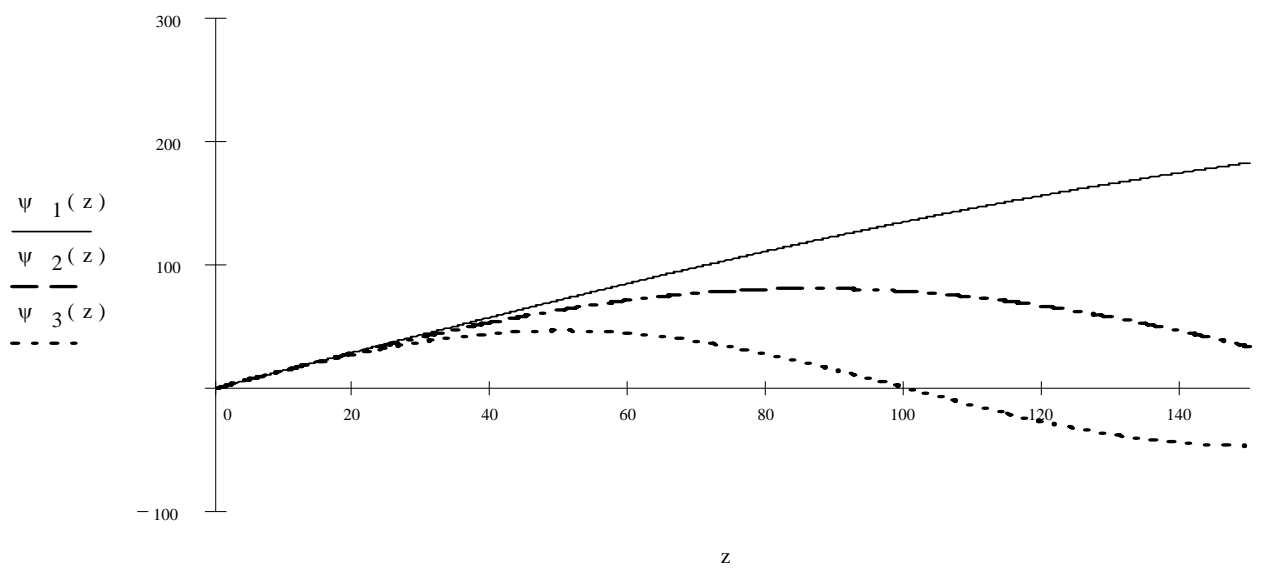

Fig. 3 Field profile in the thin film as it propagates through the thin film thickness $\left(z \times 10^{2} \mathrm{~nm}\right.$ for $\Delta \varepsilon_{p}(z)=3.50$ when $\lambda=250 \mathrm{~nm}, 650 \mathrm{~nm}$ and $950 \mathrm{~nm}$ 


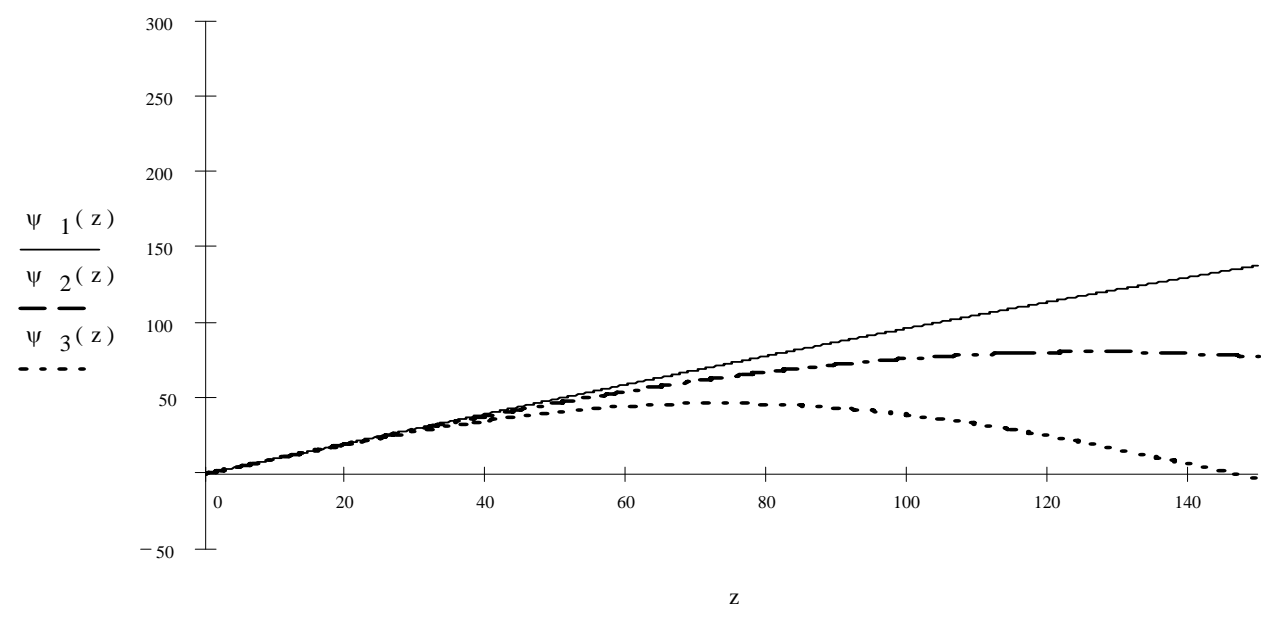

Fig.4 Field profile in the thin film as it propagates through the thin film thickness $\left(z \times 10^{2} \mathrm{~nm}\right.$ for $\Delta \varepsilon_{p}(z)=10.50$ when $\lambda=250 \mathrm{~nm}, 650 \mathrm{~nm}$ and $950 \mathrm{~nm}$

\section{CONCLUSION}

In this work we have studied the propagation of wave through a thin film medium in which dielectric perturbation was introduced as a factor affecting the propagating field profile was analyzed.W.K.B approximation technique with all the necessary boundary conditions were used as a precursor in obtaining the value of the propagating field from the defined scalar wave equation. Also considered was the effect of the variation in the value of the dielectric perturbation, $\Delta \varepsilon_{p}(z)$ on uv, optical and infrared region of the propagating electromagnetic wave through the thin film.

\section{REFERENCE}

[1] Ugwu, E.I 2011 Computation method of studying the field propagation through an inhomogeneous thin film medium using Lippmann-Schwinger Equation: Trend in Applied Science Research 6(1) 73-80.

[2] Ong H.L and Meyer.R.B 1983 Electromagnetic Wave propagation in a periodically bent Nematic liquid Crystal J.Opt Soc Am73:167-176.

[3] Abele's F 1950 Investigations on the propagation of Sinusoidal Electromagnetic wave in stratified media. Application to Thin films AnnPhy. (Daris). 5;596-640.

[4] Thyle'n, L 1983 The beam Propagation Method; An analysis of its applicability. Opt.Quantum Electron 15:433-439.

[5] Thyle' $n$ L and C. M Lee 1992 Beam Propagation method on matrix Diagonalization, J. Opt. Soc. Am. A 9(1).
[6] Feit M.D and J.A Fleck 1978 "light Propagation in graded index Optical fibers" App.Optics 17;3990-3998.

[7] Feit M.D and Fleck, J.A 1980 Computation of eigenfunctions in graded index optical fibers by the propagating Beam method App. Optic 19 2240-2246.

[8] Davydov A.S 1991 Quantum Mechanics. Pergamon pp. 451-460.

[9] Fitzpatrik, R 2005 Electromagnetic wave propagationidielectric;http://farside.ph.

utexa.edu/teaching/jkl/lectures/node79.html.

[10] Ugwu E.I 2009 Analytical study of electromagnetic wave scattering behavior using Lippmann-Schwinger equation IJPS VI.4(5) pp 310312.

[11] Ugwu, E.I and G.A Agbo 2007 Wave Propagation in non-homogeneous thin films of slowly varying refractive index W.K.B solution model PJST 8(2) 246-251.

[12] Ugwu .E.I and P. C Uduh 2005 Determination of Refractive index, Reflection /Transmittance of iron 11 sulphide thin film deposited by solution growth technique (S.G.T) JICCOTECH Maiden edition.PP.107-112.

[13] Brykhovestskii A.S and Tigrov, M. 1985; Effective Impedance Tension of the statically Rough ideally conductive Surface. Radio quantum. Electron. 703708. 